\title{
Surface Tension Reshaped Lithium Niobate Whispering Gallery Mode Micro-resonators
}

\author{
C. Y. J. Ying, ${ }^{1, *}$ G. S. Murugan, ${ }^{1}$ G. Brambilla, ${ }^{1}$ C. L. Sones, ${ }^{1}$ E. Soergel, ${ }^{2}$ \\ J. S. Wilkinson, ${ }^{1}$ R. W. Eason, ${ }^{1}$ M. N. Zervas ${ }^{1}$ and S. Mailis ${ }^{1}$ \\ ${ }^{1}$ Optoelectronics Research Centre, University of Southampton, Highfield, Southampton, SO17 1BJ, UK \\ ${ }^{2}$ Institute of Physics, University of Bonn, Wegelerstr. 8, 53115 Bonn, Germany \\ *yoy@orc.soton.ac.uk
}

\begin{abstract}
Lithium niobate micro-resonators have been fabricated by surface tension reshaping of micro-structured crystals at temperatures close to the melting point. We present preliminary results on the optical characterization of these structures.

OCIS codes: (130.3730) Lithium niobate; (070.5753) Resonators
\end{abstract}

\section{Introduction}

Lithium niobate (LN) has recently been recognized as an attractive material for high-Q whispering gallery mode (WGM) resonators due to its wide optical transparency window, high electro-optic coefficient and nonlinearity. Polishing has allowed the fabrication of large $(\sim 5 \mathrm{~mm}$ diameter $) \mathrm{LN}$ disk resonators with Q factors $>10^{8}[1]$, while microstrucuring techniques have provided the high lateral optical confinement, enabling micro-rings with small dimensions (radius $<100 \mu \mathrm{m}$ ) with $1.5-2.0 \mathrm{~nm}$ free spectral range (FSR) [2]. Recently, LN structures suitable for WGM micro-resonators have been fabricated using surface tension reshaping of previously micro-structured substrate: this method produced ultra-smooth surfaces while also maintaining the useful crystalline properties of the original material [3]. The method is based on the preferential melting of a surface layer [4] at temperatures close to the melting point for the bulk. Upon cooling the melted surface layer re-crystallizes, seeded by the bulk that remains solid during the process, and is reshaped by the surface tension to form ultra-smooth single crystal superstructures. Here we present a range of WGM structures which can be obtained as a function of the fabrication conditions (initial microstructures, thermal treatment), and some preliminary optical characterization results.

\section{Fabrication}
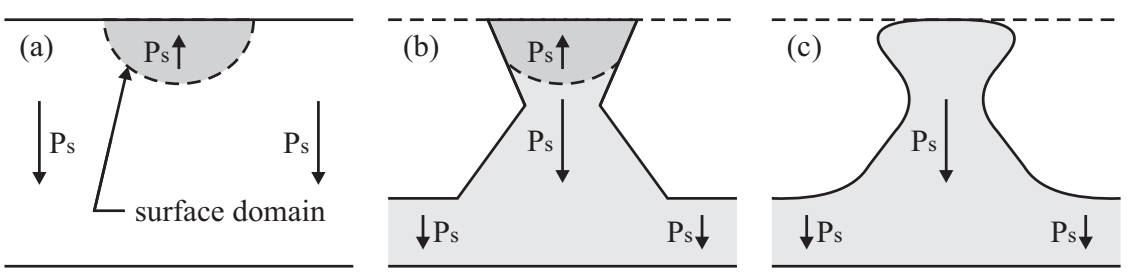

Fig. 1. Schematic of the process to fabricate LN resonators. (a): domain engineering to produce a surface domain. (b): preferential HF etching to fabricate the initial surface microstructures. (c): surface tension reshaping at temperatures close to the melting point.

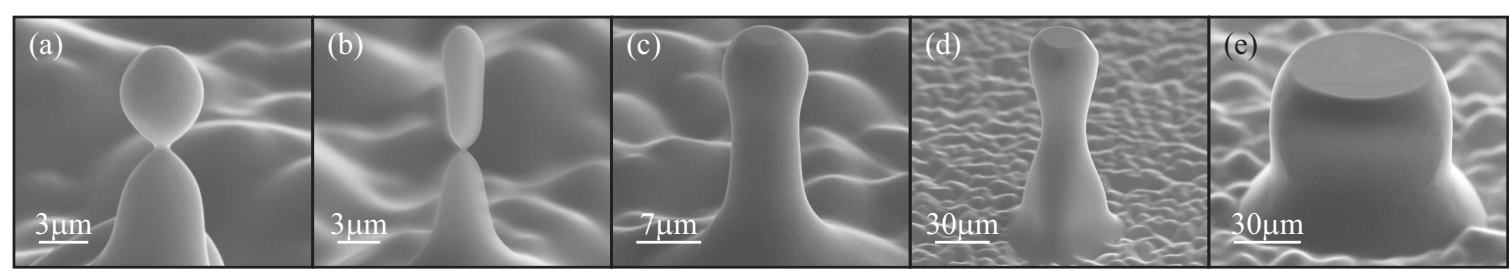

Fig. 2. SEM images tilted at $60^{\circ}$ showing a variety of surface tension reshaped structures obtained with different sizes and shapes.

The fabrication process involves: 1) domain engineering to produce inverted domain structures of limited depth, 2) HF domain orientation selective etching to produce initial surface microstructures which include an undercut as a result of the limited depth of the initial inverted domain, and 3) thermal treatment at temperatures close to the melting point to achieve preferential surface melting which allows surface tension reshaping of the initial structure to occur. The three fabrication steps are schematically illustrated in Fig. 1. Further details of the fabrication procedure can be found in [3]. Fig. 2 shows some surface tension reshaped structures, (a): $5 \mu \mathrm{m}$ prolate spheroid, (b):

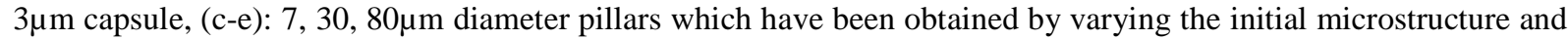
the thermal treatment conditions. It was found that there is a clear correspondence between the initial structure, thermal-treatment conditions and the final structure. Such structures are suitable for supporting WGMs as the 
smooth side surface should lead to low scattering loss. Its small dimensions can further reduce the number of supported WGMs and increase the FSR. Both features are beneficial for the fabrication of spectral filters and lasers.

\section{Optical characterisation}
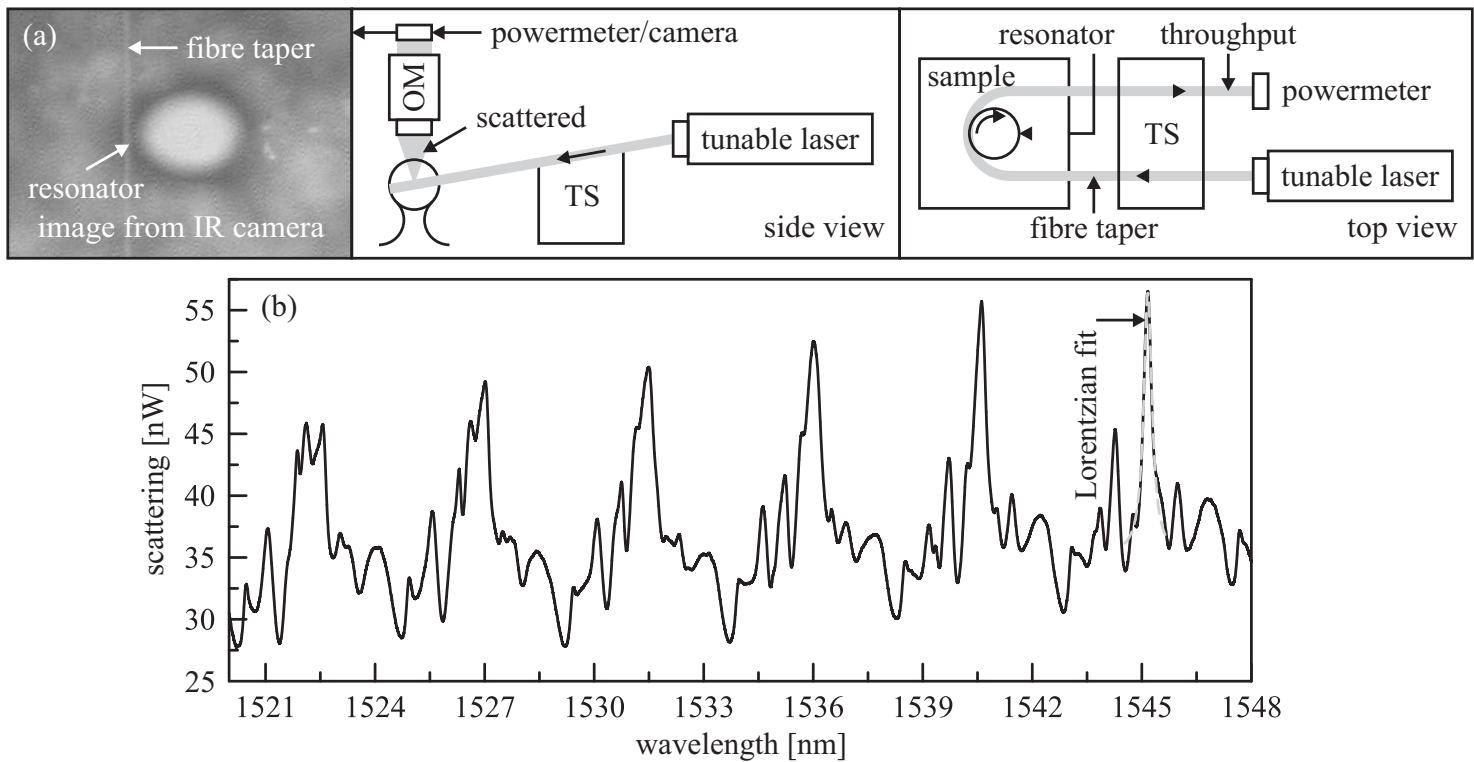

Fig. 3. (a) Schematic of the experimental configuration used to simultaneously excite and probe the optical modes in the microresonator. (TS = translation stage; OM: optical microscopy.) An optical micrograph shows the tapered optical fibre coupled to the microresonator. (b) Scattered signal with fitted Lorentzian used for the determination of Q factors of the microresonator.

Some preliminary optical characterisation of one of the surface tension reshaped WGM LN microresonators has been conducted using the structure shown in the SEM image of Fig. 2e. A schematic of the experimental configuration used to simultaneously excite and probe the optical resonances is shown in Fig. 3a. A U-shaped silica fibre taper with a waist diameter of about $2 \mu \mathrm{m}$ was used to couple light in and out of the microresonator. It was observed that when approaching the resonator the fibre taper is attracted by the surface of the structure and loads the cavity. A tunable laser source with $100 \mathrm{kHz}$ linewidth (Agilent $81600 \mathrm{~B}$ ) was used to observe the resonances of the WGM structure. In order to investigate the resonances the light transmitted through the fibre taper and the light scattered from the resonator were monitored, using InGaAs detectors, during the wavelength scanning process. The scattered light spectra are presented in Fig. 3b showing a sequence of resonances. The spectra show that we have excited more than one of the supported resonant modes. A Q factor of 5600 has been calculated by fitting a Lorentzian curve to one of the peaks in the spectrum shown in Fig. 3b with a corresponding FSR of $4.55 \mathrm{~nm}$. The shape of the resonator (Fig. 2e) resembles a truncated microbottle [5]. Such open resonators are known to leak into either side of the resonator [6], with a loss that depends on the microresonator curvature. Leakage into the resonator base was experimentally observed and it is believed to contribute to the relatively low Q. Increasing the resonator curvature will improve the resonator Q considerably.

\section{Conclusion}

Thermal treatment of micro-structured lithium niobate substrates at temperatures close to, but below the melting point, allows surface tension to reshape preferentially melted surface zones of the crystal. Preliminary optical characterization of such a single crystal micro-resonator showed a $5600 \mathrm{Q}$ factor associated with an FSR of 4.55nm.

[1] A. A. Savchenkov, V. S. Ilchenko, A. B. Matsko, and L. Maleki, "KiloHertz optical resonances in dielectric crystal cavities," Phys. Rev. A 70, 051804 (2004).

[2] A. Guarino, G. Poberaj, D. Rezzonico, R. Degl'Innocenti, and P. Günter, "Electro-optically tunable microring resonators in lithium niobate," Nat. Photon. 1, 407-410 (2007).

[3] C. Y. J. Ying, C. L. Sones, A. C. Peacock, F. Johann, E. Soergel, R. W. Eason, M. N. Zervas, and S. Mailis, "Ultra-smooth lithium niobate photonic micro-structures by surface tension reshaping," Opt. Express 18, 11508-11513 (2010).

[4] R. Lipowsky, "Surface-induced disorder and surface melting," in Springer Proc. in Physics, 1990), 158-166.

[5] G. S. Murugan, J. S. Wilkinson, and M. N. Zervas, "Selective excitation of whispering gallery modes in a novel bottle microresonator," Opt. Express 17, 11916-11925 (2009).

[6] G. S. Murugan, J. S. Wilkinson, and M. N. Zervas, "Optical excitation and probing of whispering gallery modes in bottle microresonators: potential for all-fiber add-drop filters," Opt. Lett. 35, 1893-1895 (2010). 